Pacific Journal of Mathematics

ON THE RANGE OF AN UNBOUNDED PARTLY ATOMIC 


\title{
ON THE RANGE OF AN UNBOUNDED PARTLY ATOMIC VECTOR-VALUED MEASURE
}

\author{
Enrique A. GonZÁlez-Velasco and Lee K. Jones
}

\begin{abstract}
A well-known theorem of Liapunov states that the range of a bounded, non-atomic, finite-dimensional, vector-valued measure is closed and convex. In this paper we study the range of an unbounded finite-dimensional vector-valued measure that is at least partly atomic. In the one-dimensional case we show that if the range is dense in an interval $[0, a]$ for some $a>0$ then it contains $[0, a]$. In the general case of arbitrary dimension $d$ we shall use the following notation. If $e_{1}, \ldots, e_{d}$ are linearly independent vectors in $\mathbb{R}^{d}$ let $C^{\circ}$ denote the interior of the convex cone $C=\left\{a_{1} e_{1}+\cdots+a_{d} e_{d}: a_{1}, \ldots, a_{d} \geq\right.$ $0\}$. Then, if $x=a_{1} e_{1}+\cdots+a_{d} e_{d}$ and $y=b_{1} e_{1}+\cdots+b_{d} e_{d}$ are in $C, x<y$ and $x \leq y$ shall mean that $a_{k}<b_{k}$ and that $a_{k} \leq b_{k}$, respectively, for $k=1, \ldots, d$. Finally, if $a \in C^{\circ}$ define $(0, a)=\left\{x \in C^{\circ}: 0<x<a\right\}$ and $(0, a]=\left\{x \in C^{\circ}: 0<x \leq a\right\}$. Now let $\mu$ be a measure such that any bounded subset of its range is in $C$, and such that the set of all $\mu(E)$ in $C$ such that $E$ contains no atom is bounded. We show that if $R_{\mu}$ is dense in $(0, a]$ for some $a \in C^{\circ}$ then it contains $(0, a]$.
\end{abstract}

A well-known theorem of Liapunov [1] states that the range of a bounded, non-atomic, finite-dimensional vector measure is closed and convex. In the case of an unbounded measure the range remains convex but need not be closed. Additional properties of the range in this case have been studied by $\mathrm{C}$. Olech [2]. When a measure is at least partly atomic, one cannot hope to extend these results even in the onedimensional case. There is, however, an extension when the range of the measure is dense, and this result appears to be non-trivial for an unbounded measure. Our proof will include the easy case when the measure is bounded.

Furthermore, a typical argument in showing that a linear operator has an inverse is to show first that it has a dense range and then conclude, via a closed range theorem, that its range is closed. It is natural to ask to what extent this type of argument holds for not necessarily linear but simply additive mappings. A measure is one of the fundamental structures in mathematics. It is not linear but it is additive on disjoint subsets. The results in this paper give conditions under which we can conclude that if the range of a finite-dimensional vector 
measure is dense then it is closed.

We consider first the one-dimensional case. Let $(S, \mathscr{M}, \mu)$ be a positive measure, that is, a space $S$, a $\sigma$-algebra $\mathscr{M}$ of subsets of $S$ and a countably additive function $\mu: \mathscr{M} \rightarrow[0, \infty]$, and let $R_{\mu}$ denote its range. Notice that we allow $R_{\mu}$ to contain $\infty$.

THEOREM 1. Let $\mu$ be a positive measure. If $R_{\mu}$ is dense in $[0, a]$ for some $a>0$ then it contains $[0, a]$.

Proof. It is enough to prove it for a $\sigma$-finite measure. Otherwise, consider a countable collection of elements of $\mathscr{M}$ whose measures are dense in $[0, a]$. These elements generate a $\sigma$-finite measure, and if its range contains $[0, a]$ so does $R_{\mu}$.

Now, let $R_{A}$ and $R_{N}$ denote the ranges of the atomic and nonatomic components of $\mu$, respectively, and for any $x \in(0, a]$ define

$$
\mathscr{A}_{x}=\{A \in \mathscr{M}: A \text { is an atom and } \mu(A) \in(0, x)\},
$$

and let $x_{0} \in(0, a]$. We shall assume that $x_{0} \notin R_{\mu}$ and arrive at a contradiction. Consider first the case in which $\mathscr{A}_{x_{0}}$ is not $\mu$-summable, which we define to mean that the measures of its elements do not have a finite sum. Then there is a positive integer $p_{1}$ and disjoint atoms $A_{1}^{1}, \ldots, A_{p_{1}+1}^{1} \in \mathscr{A}_{x_{0}}$ with $\mu\left(A_{p_{1}+1}^{1}\right) \leq \cdots \leq \mu\left(A_{1}^{1}\right)$ such that

$$
\frac{1}{2} x_{0} \leq \sum_{i=1}^{p_{1}} \mu\left(A_{i}^{1}\right)<x_{0}<\sum_{i=1}^{p_{1}+1} \mu\left(A_{i}^{1}\right)
$$

and

$$
0<x_{1} \stackrel{\text { def }}{=} x_{0}-\sum_{i=1}^{p_{1}} \mu\left(A_{i}^{1}\right) \leq \frac{1}{2} x_{0} .
$$

Notice that $x_{1}<\mu\left(A_{i}^{1}\right)$ for $i=1, \ldots, p_{1}$. If $\mathscr{A}_{x_{1}}$ is not $\mu$-summable, we can restart the process with $x_{1}$ in place of $x_{0}$, and this can be done again if possible. At the $n$th stage, $n \geq 1$, we have a positive integer $p_{n}$, and disjoint atoms $A_{1}^{n}, \ldots, A_{p_{n}+1}^{n} \in \mathscr{A}_{x_{n-1}}$, which are automatically disjoint from those chosen at any previous stage, such that $\mu\left(A_{p_{n}+1}^{n}\right) \leq \cdots \leq \mu\left(A_{1}^{n}\right)$,

$$
\begin{gathered}
\frac{1}{2} x_{n-1} \leq \sum_{i=1}^{p_{n}} \mu\left(A_{i}^{n}\right)<x_{n-1}<\sum_{i=1}^{p_{n}+1} \mu\left(A_{i}^{n}\right), \\
0<x_{n} \stackrel{\text { def }}{=} x_{n-1}-\sum_{i=1}^{p_{n}} \mu\left(A_{i}^{n}\right)=x_{0}-\sum_{j=1}^{n} \sum_{i=1}^{p_{j}} \mu\left(A_{i}^{j}\right) \leq \frac{1}{2} x_{n-1},
\end{gathered}
$$


and $x_{n}<\mu\left(A_{i}^{j}\right)$ for $i=1, \ldots, p_{n}$ and $j=1, \ldots, n$. If this process can be repeated indefinitely then the $x_{n}$ form an infinite sequence that, by construction, converges to zero, and then

$$
x_{0}=\sum_{j=1}^{\infty} \sum_{i=1}^{p_{j}} \mu\left(A_{i}^{j}\right)=\mu\left(\bigcup_{j=1}^{\infty} \bigcup_{i=1}^{p_{j}} A_{i}^{j}\right) \in R_{A} \subset R_{\mu},
$$

contradicting the original assumption.

Now, if $\mathscr{A}_{x_{n}}$ is $\mu$-summable for some $n \geq 0$, then the restriction of $R_{A}$ to $\left[0, x_{n}\right]$ is a closed set and, since the restriction of $R_{N}$ to $\left[0, x_{n}\right]$ is also closed, it follows that the restriction of $R_{\mu}$ to $\left[0, x_{n}\right]$, which is the sum of the two previous restrictions restricted to $\left[0, x_{n}\right]$, is a closed set. Since it is also dense, there are sets $A$ and $E$ in such that $A$ is either empty or a union of atoms disjoint from the $A_{i}^{j}$, $E$ contains no atom, and $x_{n}=\mu(A)+\mu(E)$. Therefore,

$$
\begin{aligned}
x_{0} & =\mu(A)+\mu(E)+\sum_{j=1}^{n} \sum_{i=1}^{p_{j}} \mu\left(A_{i}^{j}\right) \\
& =\mu\left[A \cup E \cup\left(\bigcup_{j=1}^{n} \bigcup_{i=1}^{p_{j}} A_{i}^{j}\right)\right] \in R_{\mu},
\end{aligned}
$$

contradicting, again, the original assumption.

It should be remarked that this theorem does not hold if the measure is not positive. For if $\mu$ is purely atomic and the measures of its atoms are -1 and all the rational numbers in $[1, \infty)$ then its range is dense in $[0,1]$ but does not contain $[0,1]$.

To deal with the general case of arbitrary dimension $d$ we develop some additional notation and terminology. If $e_{1}, \ldots, e_{d}$ are linearly independent vectors in $\mathbb{R}^{d}$ consider the convex cone

$$
C=\left\{x=a_{1} e_{1}+\cdots+a_{d} e_{d}: a_{1}, \ldots, a_{d} \geq 0\right\},
$$

let $C^{\circ}$ denote the interior of $C$, and if $x=a_{1} e_{1}+\cdots+a_{d} e_{d}$ and $y=b_{1} e_{1}+\cdots+b_{d} e_{d}$ are in $C, x<y$ and $x \leq y$ shall mean that $a_{k}<b_{k}$ and that $a_{k} \leq b_{k}$, respectively, for $k=1, \ldots, d$. If $a, b \in$ $C$ and $a<b$ define

$$
(a, b)=\left\{x \in C^{\circ}: a<x<b\right\}
$$

and

$$
(a, b]=\left\{x \in C^{\circ}: a<x \leq b\right\}
$$


Now, let $(S, \mathscr{M}, \mu)$ be a vector-valued measure in $C$, that is, a space $S$, a $\sigma$-algebra $\mathscr{M}$ of subsets of $S$ and a countably additive function $\mu$ defined on $\mathscr{M}$ and taking values in $C$ or infinity. Infinity is defined as any linear combination of the $e_{k}$ in which at least one coefficient is $+\infty$. Let $R_{\mu}$ denote the range of $\mu$.

THEOREM 2. Let $\mu$ be a vector-valued measure in $C$ such that the set of all $\mu(E) \in C$ such that $E$ contains no atom is bounded. If $R_{\mu}$ is dense in $(0, a]$ for some $a \in C^{\circ}$ then it contains $(0, a]$.

Proof. As in the one-dimensional case, it is enough to prove it for a $\sigma$-finite measure, and again we denote by $R_{A}$ and $R_{N}$ the ranges of its atomic and non-atomic components. For simplicity we carry out the proof in the two-dimensional case, and then indicate how it is to be modified in the general case.

If $x=a_{1} e_{1}+a_{2} e_{2}$ is in $C$, let $0 x$ denote the segment $\{t x: 0<t \leq$ $1\}$ and, for $k=1,2$, let $x^{k}$ and $C_{k x}$ denote the vector $a_{k} e_{k}$ and the closed triangle with vertices $0, x$ and $x^{k}$, respectively. Finally, define

$$
\mathscr{A}_{k x}=\left\{A \in \mathscr{M}: A \text { is an atom and } \mu(A) \in C_{k x} \cap(0, x)\right\}
$$

and define $y \in C$ to be a $\mu$-cluster point for $\mathscr{A}_{k x}$ if for every open disc $D \subset \mathbb{R}^{2}$ centered at $y$ there are infinitely many atoms in $\mathscr{A}_{k x}$ whose measures are in $D \cap C$.

Now, let $x_{0} \in(0, a]$ be arbitrary and assume first that for any $x \in 0 x_{0}$ the sets $\mathscr{A}_{1 x}$ and $\mathscr{A}_{2 x}$ are not $\mu$-summable. Then there is a positive integer $p_{1}$ and disjoint atoms $A_{1}^{1}, \ldots, A_{p_{1}}^{1}$ such that

$$
\frac{1}{2} x_{0} \leq \sum_{i=1}^{p_{1}} \mu\left(A_{i}^{1}\right)<x_{0}
$$

and

$$
0<x_{1} \stackrel{\text { def }}{=} x_{0}=\sum_{i=1}^{p_{1}} \mu\left(A_{i}^{1}\right) \leq \frac{1}{2} x_{0} .
$$

If for any $x \in 0 x_{1}$ the sets $\mathscr{A}_{1 x}$ and $\mathscr{A}_{2 x}$ are not $\mu$-summable, we can restart the process with $x_{1}$ in place of $x_{0}$, and this can be done again if possible. At the $n$th stage, $n \geq 1$, we have a positive integer $p_{n}$ and disjoint atoms $A_{1}^{n}, \ldots, A_{p_{n}}^{n}$, which are disjoint from those chosen at any previous stage, such that

$$
\frac{1}{2} x_{n-1} \leq \sum_{i=1}^{p_{n}} \mu\left(A_{i}^{n}\right)<x_{n-1}
$$


and

$$
0<x_{n} \stackrel{\text { def }}{=} x_{n-1}-\sum_{i=1}^{p_{n}} \mu\left(A_{i}^{n}\right)=x_{0}-\sum_{j=1}^{n} \sum_{i=1}^{p_{j}} \mu\left(A_{i}^{j}\right) \leq \frac{1}{2} x_{n-1} .
$$

If this process can be repeated indefinitely then the $x_{n}$ form an infinite sequence that, by construction, converges to the origin, and then

$$
x_{0}=\sum_{j=1}^{\infty} \sum_{i=1}^{p_{j}} \mu\left(A_{i}^{j}\right)=\mu\left(\bigcup_{j=1}^{\infty} \bigcup_{i=1}^{p_{j}} A_{i}^{j}\right) \in R_{A} \subset R_{\mu} .
$$

If, on the other hand, there is an $n \geq 0$, a point $x \in 0 x_{n}$ and a value of $k$, which we assume to be $k=1$, such that $\mathscr{A}_{1 x}$ is $\mu$-summable, let $x_{c}$ denote either $x_{n}$ if $\mathscr{A}_{1 x_{n}}$ is $\mu$-summable or, otherwise, the closest point to the origin in $0 x_{n}$ with the property that the closed segment from $x_{c}$ to $x_{c}^{1}$ contains a $\mu$-cluster point for $\mathscr{A}_{1 x_{n}}$. In the second case there is a positive integer $q_{0}$ and disjoint atoms $B_{1}^{0}, \ldots, B_{q_{0}}^{0} \in \mathscr{A}_{1 x_{n}}$, which are disjoint from those chosen at any previous stage, such that if

$$
y_{0} \stackrel{\text { def }}{=} x_{n}-\sum_{i=1}^{q_{0}} \mu\left(B_{i}^{0}\right)
$$

then $y_{0}^{1} \in 0 x_{c}^{1}$ and, in addition, $y_{0}^{1}<x_{c}^{1}$ if $\mathscr{A}_{1 x_{c}}$ is not $\mu$-summable. In the first case, when $\mathscr{A}_{1 x_{n}}$ is $\mu$-summable, define $y_{0}=x_{c}=x_{n}$ and take $B_{i}^{0}$ to be the empty set for each $i$ so that the equation above remains valid. Then choose $x \in 0 x_{c}$ such that $\mathscr{A}_{1 x}$ is $\mu$-summable and $y_{0}^{1} \in 0 x^{1}$, and notice that the set $\Sigma_{1}$ of all sums of measures of elements of $\mathscr{A}_{1 x}$ is closed and disjoint from $0 x^{1}$.

Now, if $\mathscr{A}_{2 y_{0}}$ is not $\mu$-summable, then there is a positive integer $q_{1}$ and disjoint atoms $B_{1}^{1}, \ldots, B_{q_{1}}^{1} \in \mathscr{A}_{2 y_{0}}$, which are disjoint from those chosen at any previous stage, such that

$$
0<y_{1} \stackrel{\text { def }}{=} y_{0}-\sum_{i=1}^{q_{1}} \mu\left(B_{i}^{1}\right)
$$

and $y_{1}^{2} \leq y_{0}^{2} / 2$. This process can now be repeated as many times as possible. At the $m$ th stage, $m \geq 1$, we have a positive integer $q_{m}$ and disjoint atoms $B_{1}^{m}, \ldots, B_{q_{m}}^{m} \in \mathscr{A}_{2 y_{m-1}}$, which are disjoint from those chosen at any previous stage, such that

$$
0<y_{m} \stackrel{\text { def }}{=} y_{m-1}-\sum_{i=1}^{q_{m}} \mu\left(B_{i}^{m}\right)
$$


and $y_{m}^{2} \leq y_{m-1}^{2} / 2$. If this process can be repeated indefinitely then the $y_{m}$ form a sequence that, by construction, converges to some point $s_{1} \in\{0\} \cup 0 x^{1}$. Next we claim that the restriction of $R_{\mu}$ to $0 x^{1}$ is dense in $0 x^{1}$. In fact, every point $s \in 0 x^{1}$ is the limit of a sequence $\left\{s_{k}\right\}$ in $(0, a]$ where each $s_{k}$ is the sum of four elements: $s_{k 1} \in\{0\} \cup \Sigma_{1}, s_{k 2} \in C_{2 x}, s_{k 3} \in R_{N}$ and $s_{k 4}$, which is either 0 or is in the restriction of $R_{A}$ to $0 x^{1}$. But $s_{k 1} \rightarrow 0$ and $s_{k 2} \rightarrow 0$ as $k \rightarrow \infty$, and, by considering a subsequence if necessary, $s_{k 3}$ converges to some $s_{N} \in R_{N}$, since this last set is closed by Liapunov's theorem. Notice that $s_{N} \in\{0\} \cup 0 x^{1}$, and then $s_{N}+s_{k 4}$ is arbitrarily close to $s$ for $k$ large enough. This proves the claim and then, by Theorem 1 , the restriction of $R_{\mu}$ to $0 x^{1}$ contains $0 x^{1}$. It follows that $s_{1}=$ $\mu\left(E_{1}\right)$ where $E_{1} \in \mathscr{M}$ is disjoint from any of the atoms chosen above. Therefore,

$$
\begin{aligned}
x_{0} & =x_{n}+\sum_{j=1}^{n} \sum_{i=1}^{p_{j}} \mu\left(A_{i}^{j}\right) \\
& =\mu\left(E_{1}\right)+\sum_{m=0}^{\infty} \sum_{i=1}^{q_{m}} \mu\left(B_{i}^{m}\right)+\sum_{j=1}^{n} \sum_{i=1}^{p_{j}} \mu\left(A_{i}^{j}\right) \\
& =\mu\left[E_{1} \cup\left(\bigcup_{m=0}^{\infty} \bigcup_{i=1}^{q_{m}} B_{i}^{m}\right) \cup\left(\bigcup_{j=1}^{n} \bigcup_{i=1}^{p_{j}} A_{i}^{j}\right)\right] \in R_{\mu} .
\end{aligned}
$$

If the process described above cannot be repeated indefinitely, then there is an $m \geq 0$ such that, $\mathscr{A}_{2 y_{m}}$ is $\mu$-summable. Then the set $\Sigma_{2}$ of all sums of elements of $\mathscr{A}_{2 y_{m}}$ is closed, and an argument like the one above applied to $y_{m}^{1}$ and $y_{m}^{2}$ shows that $y_{m}=\mu\left(E_{1}\right)+\mu\left(E_{2}\right)$, where $E_{1}$ and $E_{2}$ are disjoint from each other and from any previously chosen atoms. In this case

$$
\begin{aligned}
x_{0} & =x_{n}+\sum_{j=1}^{n} \sum_{i=1}^{p_{j}} \mu\left(A_{i}^{j}\right) \\
& =\mu\left(E_{1}\right)+\mu\left(E_{2}\right)+\sum_{j=0}^{m} \sum_{i=1}^{q_{m}} \mu\left(B_{i}^{m}\right)+\sum_{j=1}^{n} \sum_{i=1}^{p_{j}} \mu\left(A_{i}^{j}\right) \\
& =\mu\left[E_{1} \cup E_{2} \cup\left(\bigcup_{j=0}^{m} \bigcup_{i=1}^{q_{m}} B_{i}^{m}\right) \cup\left(\bigcup_{j=1}^{n} \bigcup_{i=1}^{p_{j}} A_{i}^{j}\right)\right] \in R_{\mu} .
\end{aligned}
$$

This completes the proof in the two-dimensional case. 
The same proof is valid in the general case with the following changes. Let $H_{k x}$ denote the hyperplane of codimension one consisting of all points of the form $x^{k}+\sum_{j \neq k} a_{j} e_{j}, a_{j} \in \mathbb{R}$, and let $C_{k x}$ denote now the closed convex hull of $\{0\} \cup\left(H_{k x} \cap(0, x]\right)$. Then the segment from $x_{c}$ to $x_{c}^{1}$ above is replaced with the set of all points in $H_{1 x_{c}}$ that are in the closure of $C_{1 x_{c}}$. With these changes the previous proof is valid until the definition of $y_{0}$. The $y_{m}$ are also defined as above, if $\mathscr{A}_{k y_{m-1}}$ is not $\mu$-summable for each $k \geq 2$, but the $B_{i}^{m}$ are chosen from $\bigcup_{k=2}^{d} \mathscr{A}_{k y_{m-1}}$ and so that $y_{m}^{k} \leq y_{m-1}^{k} / 2$ for $k \geq 2$. If the $y_{m}$ form an infinite sequence that converges to a point $s_{1} \in\{0\} \cup 0 x^{1}$, the previous proof is still valid. Otherwise, one or more of the $\mathscr{A}_{k y_{m}}$ become $\mu$-summable along the way. It should be clear how the components of $y_{m}$ that do not become zero are in $R_{\mu}$ and how this can be done using sets disjoint from all others.

It is not possible to assert that $R_{\mu}$ contains $[0, a]$, for if $\mu$ is purely atomic and the measures of its atoms are the points with positive rational coordinates in $(0,1)$ then $R_{\mu}$ does not contain any point on the coordinate axes except the origin.

\section{REFERENCES}

[1] A. A. Liapunov, Sur les fonctions-vecteurs complètement additives, Izv. Akad. Nauk SSSR, Ser. Mat., 8 (1940), 465-478.

[2] C. Olech, On the range of an unbounded vector-valued measure, Math. Systems Theory, 2 (1968), 251-256.

Received December 3, 1990 and in revised form March 12, 1991.

UNIVERSITY OF MASSACHUSETtS

LOWELL, MA 01854 



\section{PACIFIC JOURNAL OF MATHEMATICS EDITORS}

\author{
V. S. VARADARAJAN \\ (Managing Editor) \\ University of California \\ Los Angeles, CA 90024-1555 \\ Herbert Clemens \\ University of Utah \\ Salt Lake City, UT 84112 \\ F. Michael Christ \\ University of California \\ Los Angeles, CA 90024-1555 \\ THOMAS ENRIGHT \\ University of California, San Diego \\ La Jolla, CA 92093
}

\author{
Nicholas ERcolani \\ University of Arizona \\ Tucson, AZ 85721 \\ R. FINN \\ Stanford University \\ Stanford, CA 94305 \\ VAughan F. R. JONES \\ University of California \\ Berkeley, CA 94720 \\ C. C. Moore \\ University of California \\ Berkeley, CA 94720 \\ MARTIN ScharlemanN \\ University of California \\ Santa Barbara, CA 93106 \\ Harold Stark \\ University of California, San Diego \\ La Jolla, CA 92093
}

STEVEN KeRCKHOFF

Stanford University

Stanford, CA 94305

\begin{tabular}{|c|c|c|c|c|}
\hline \multicolumn{5}{|c|}{ ASSOCIATE EDITORS } \\
\hline R. Arens & $\begin{array}{ll}\text { E. F. BECKENBACH } & \text { B. H } \\
(1906-1982) & \end{array}$ & NeUmanN & $\begin{array}{c}\text { F. WoLF } \\
(1904-1989)\end{array}$ & K. Yoshida \\
\hline \multicolumn{5}{|c|}{ SUPPORTING INSTITUTIONS } \\
\hline \multicolumn{2}{|c|}{ UNIVERSITY OF ARIZONA } & \multicolumn{3}{|c|}{ UNIVERSITY OF OREGON } \\
\hline \multicolumn{2}{|c|}{ UNIVERSITY OF BRITISH COLUMBIA } & \multicolumn{3}{|c|}{ UNIVERSITY OF SOUTHERN CALIFORNIA } \\
\hline \multicolumn{2}{|c|}{ CALIFORNIA INSTITUTE OF TECHNOLOGY } & \multicolumn{3}{|c|}{ STANFORD UNIVERSITY } \\
\hline \multicolumn{2}{|c|}{ UNIVERSITY OF CALIFORNIA } & \multicolumn{3}{|c|}{ UNIVERSITY OF HAWAII } \\
\hline \multicolumn{2}{|c|}{ MONTANA STATE UNIVERSITY } & \multicolumn{3}{|c|}{ UNIVERSITY OF TOKYO } \\
\hline \multicolumn{2}{|c|}{ UNIVERSITY OF NEVADA, RENO } & \multicolumn{3}{|c|}{ UNIVERSITY OF UTAH } \\
\hline & \multicolumn{3}{|c|}{ WASHINGTON STATE UNIVERSITY } \\
\hline \multicolumn{2}{|c|}{ OREGON STATE UNIVERSITY } & \multicolumn{3}{|c|}{ UNIVERSITY OF WASHINGTON } \\
\hline
\end{tabular}

The Supporting Institutions listed above contribute to the cost of publication of this Journal, but they are not owners or publishers and have no responsibility for its content or policies.

Mathematical papers intended for publication in the Pacific Journal of Mathematics should be in typed
form or offset-reproduced (not dittoed), double spaced with large margins. Please do not use built up fractions
in the text of the manuscript. However, you may use them in the displayed equations. Underline Greek letters
in red, German in green, and script in blue. The first paragraph must be capable of being used separately as
a synopsis of the entire paper. In particular it should contain no bibliographic references. Please propose a
heading for the odd numbered pages of less than 35 characters. Manuscripts, in triplicate, may be sent to any
one of the editors. Please classify according to the 1991 Mathematics Subject Classification scheme which
can be found in the December index volumes of Mathematical Reviews. Supply name and address of author
to whom proofs should be sent. All other communications should be addressed to the managing editor, or
Elaine Barth, University of California, Los Angeles, California $90024-1555-05$.
There are page-charges associated with articles appearing in the Pacific Journal of Mathematics. These
charges are expected to be paid by the author's University, Government Agency or Company. If the author or
authors do not have access to such Institutional support these charges are waived. Single authors will receive
50 free reprints; joint authors will receive a total of 100 free reprints. Additional copies may be obtained at
cost in multiples of 50 .

The Pacific Journal of Mathematics (ISSN 0030-8730) is published monthly except for July and August. Regular subscription rate: $\$ 190.00$ a year (10 issues). Special rate: $\$ 95.00$ a year to individual members of supporting institutions.

Subscriptions, orders for numbers issued in the last three calendar years, and changes of address should be sent to Pacific Journal of Mathematics, P.O. Box 969, Carmel Valley, CA 93924, U.S.A. Old back numbers obtainable from Kraus Periodicals Co., Route 100, Millwood, NY 10546.

The Pacific Journal of Mathematics at P.O. Box 969, Carmel Valley, CA 93924 (ISSN 0030-8730) is published monthly except for July and August. Second-class postage paid at Carmel Valley, California 93924, and additional mailing offices. Postmaster: send address changes to Pacific Journal of Mathematics, P.O. Box 969, Carmel Valley, CA 93924.

PUBLISHED BY PACIFIC JOURNAL OF MATHEMATICS, A NON-PROFIT CORPORATION Copyright (C) 1992 by Pacific Journal of Mathematics 


\section{Pacific Journal of Mathematics}

\section{Vol. 154, No. $2 \quad$ June, 1992}

Manuel (Rodriguez) de León, J. A. Oubiña, P. R. Rodrigues and

Modesto R. Salgado, Almost $s$-tangent manifolds of higher order . . . . 201

Martin Engman, New spectral characterization theorems for $S^{2} \ldots \ldots \ldots 215$

Yuval Zvi Flicker, The adjoint representation $L$-function for GL $(n) \ldots \ldots 231$

Enrique Alberto Gonzalez-Velasco and Lee Kenneth Jones, On the range

of an unbounded partly atomic vector-valued measure . ............ 245

Takayuki Hibi, Face number inequalities for matroid complexes and

Cohen-Macaulay types of Stanley-Reisner rings of distributive

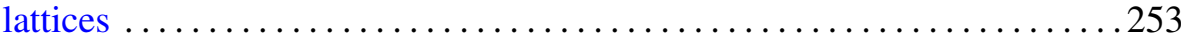

Hervé Jacquet and Stephen James Rallis, Kloosterman integrals for skew

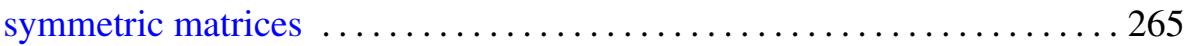

Shulim Kaliman, Two remarks on polynomials in two variables .........285

Kirk Lancaster, Qualitative behavior of solutions of elliptic free boundary problems ..........................................297

Feng Luo, Actions of finite groups on knot complements . ........... 317

James Joseph Madden and Charles Madison Stanton, One-dimensional

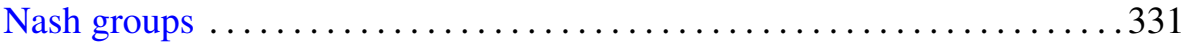

Christopher K. McCord, Estimating Nielsen numbers on

infrasolvmanifolds ......................................... 345

Gordan Savin, On the tensor product of theta representations of $\mathrm{GL}_{3}$ 369

Gerold Wagner, On means of distances on the surface of a sphere. II.

(Upper bounds) 